\title{
TAME NEAR-RINGS AND N-GROUPS
}

\author{
by S. D. SCOTT
}

(Received 4th December 1978)

Throughout this paper any near-ring $N$ will be left distributive, zero symmetric and have an identity. Furthermore, all $N$-groups will be unitary. All groups considered will be written additively. This does not imply commutativity.

Suppose $V$ is a group and $S$ a set of endomorphisms of $V$ containing the inner automorphisms. Let $N$ be the near-ring of maps of $V$ into $V$ generated by $S$. It is easily seen that $V$ is a unitary $N$-group. Suppose $W$ is an $N$-subgroup of $V$. Since $W S \subseteq W, W$ is a normal subgroup and, by $(6,6.6, \mathrm{p} .174), W$ is a submodule of $V$ i.e. the kernel of an $N$-homomorphism on $V$.

This leads to a definition. Let $V$ be an $N$-group. We call $V$ tame if every $N$-subgroup of $V$ is a submodule. A near-ring $N$ with a faithful tame $N$-group will be called tame.

Tame near-rings and $N$-groups were first investigated by the author in his Ph.D. dissertation (7, Chs 4, 5 \& 6). Many of the results of this paper may be found in (7), although the proofs given here are often simpler.

The example given above has a much stronger property than tameness. In Section six 2 -tame and compatible $N$-groups are defined and these are looked at in more detail in subsequent sections.

\section{Nilpotency in tame near-rings}

If $S$ is a subset of a near-ring $N$, then $R(S)$ will denote the right ideal of $N$ generated by $S$. With the aid of certain propositions and lemmas we investigate nilpotency in tame near-rings.

Proposition 1.1. Let $N$ be a near-ring and $V$ a tame $N$-group. If $M$ is a right $N$-subgroup of $N$, then $v R(M)=v M$ for all $v$ in $V$.

Proof. Since, for $v$ in $V, v M$ is a submodule of $V,(v M: v)$ is a right ideal of $N$. Clearly $M \leqq(v M: v), R(M) \leqq(v M: v)$ and $v R(M) \leqq v M$. The result now follows.

Lemma 1.2. Let $N$ be a tame near-ring, $M$ a right $N$-subgroup of $N$, and $S_{i}, i=1,2$, non-empty subsets of $N$. If one of the subsets $S_{1} M, S_{1} M S_{2}$ or $M S_{2}$ of $N$ is $\{0\}$, then the same applies on replacing $M$ by $R(M)$. 
Proof. If $S_{1} M=\{0\}$, then $M \leqq\left(0: S_{1}\right), R(M) \leqq\left(0: S_{1}\right)$ and $S_{1} R(M)=\{0\}$. Suppose that $S_{1} M S_{2}=\{0\}$ and $V$ is a faithful tame $N$-group. By 1.1 it follows that for all $\alpha$ in $S_{1}, \beta$ in $S_{2}$ and $v$ in $V$

$$
v \alpha R(M) \beta=v \alpha M \beta=\{0\} .
$$

Thus $V S_{1} R(M) S_{2}=\{0\}$ and $S_{1} R(M) S_{2}=\{0\}$. Taking $S_{1}=\{1\}$ the case where $M S_{2}=\{0\}$ follows.

Proposition 1.3. Let $N$ be a tame near-ring and $H_{1}, \ldots, H_{k}$, right $N$-subgroups of $N$ for $i=1, \ldots, k$. If $H_{1} H_{2} \ldots H_{k}=\{0\}$, then

$$
R\left(H_{1}\right) R\left(H_{2}\right) \ldots R\left(H_{k}\right)=\{0\} .
$$

Proof. This follows by repeated application of 1.2.

Corollary. If $M$ is a nilpotent right $N$-subgroup of a tame near-ring $N$, then $R(M)$ is nilpotent.

Theorem 1.4. If $N$ is a tame near-ring and $A$ the sum of all nilpotent right ideals of $N$, then $A$ is an ideal of $N$.

Proof. We have $A=\sum_{i \in I} R_{i}$, where $\left\{R_{i}, i \in \eta\right\}$ is the set of all nilpotent right ideals of $N$. Thus for any $\alpha$ in $N$

$$
\alpha A=\sum_{i \in I} \alpha R_{i}
$$

and each $\alpha R_{i}$ is a nilpotent right $N$-subgroup of $N$. By the above corollary each $R\left(\alpha R_{i}\right)$ is nilpotent. Thus for each $\alpha$ in $N$

$$
\alpha A \leqq \sum_{i \in I} R\left(\alpha R_{i}\right) \leqq A
$$

and it follows that $A$ is an ideal.

We note that there exist near-rings where the sum of all nilpotent right ideals need no longer be an ideal (see (1, p. 204)) but, as 1.4 indicates, tame near-rings resemble rings more closely. Further evidence of this similarity is seen in many of the results that follow.

\section{Direct sums}

Let $N$ be a near-ring with an $N$-group $V$. It may happen that $N=A \oplus B$ where $A$ and $B$ are ideals, but this split in $N$ does not induce a corresponding split in $V$ (e.g. if $p$ and $q$ are distinct primes, then $\boldsymbol{Z}_{p q}=\boldsymbol{Z}_{p} \oplus \boldsymbol{Z}_{q}$ and any group of exponent $p q$ is a faithful unitary $\boldsymbol{Z}_{p q}$-group). However, for tame $N$-groups the situation is simpler.

In order to investigate this situation we need an alternative characterisation of tame. The following proposition provides this characterisation which will be of importance in defining 2-tame, and compatible $N$-groups. 
Proposition 2.1. An $N$-group $V$ is tame if, and only if, for $v$ and $w$ in $V$ and $\alpha$ in $N$ there exists $\beta$ in $N$ such that

$$
(v+w) \alpha-v \alpha=w \beta .
$$

Proof. If $V$ is tame, then $w N$ is a submodule of $V$ containing $w$. Thus $(v+w) \alpha-v \alpha$ is in $w N$ and $\beta$ exists.

Suppose for given $v, w$ and $\alpha, \beta$ exists. Let $W$ be an $N$-subgroup of $V$. If $w$ is in $W$ clearly $(v+w) \alpha-v \alpha$ is in $W$. Thus $W$ is a submodule of $V$ (normality follows on taking $\alpha=1$ ). The proposition is proved.

Theorem 2.2. Let $N$ be a near-ring with a faithful tame $N$-group $V$. If $N=A \oplus B$ where $A$ and $B$ are ideals of $N$, then $V=U \oplus W$ with $U$ and $W$ submodules of $V$ such that $(0: W)=A$ and $(0: U)=B$.

Proof. We have $1=e_{1}+e_{2}$ where $e_{1}$ is in $A$ and $e_{2}$ is in $B$. The $e_{i}, i=1,2$, are central orthogonal idempotents.

Define $U$ to be the subset $V e_{1}$ of $V$ and $W$ the subset $V e_{2}$ of $V$. Now $U=$ $\left\{v \in V: v e_{2}=0\right\}$, since $U e_{2}=\{0\}$ and, if $v e_{2}=0$ for some $v$ in $V$, then $v=v e_{1}+v e_{2}=v e_{1}$. To show $U$ is a submodule of $V$ it is sufficient to show that it is an $N$-subgroup. Now

$$
U N=V e_{1} N=V N e_{1} \subseteq U
$$

since $e_{1}$ is central. In particular $u(-1)=-u$ is in $U$ for all $u$ in $U$. We must therefore show that $U$ is closed under addition. Let $v_{1} e_{1}$ and $v_{2} e_{1}$ be elements of $U$. By 2.1

$$
\left(v_{1} e_{1}+v_{2} e_{1}\right) e_{2}-v_{1} e_{1} e_{2}=v_{2} e_{1} \beta
$$

for some $\beta$ in $N$. Now $v_{1} e_{1} e_{2}=0$ and

Hence

$$
\left(v_{1} e_{1}+v_{2} e_{1}\right) e_{2}=v_{2} e_{1} \beta \text {. }
$$

$$
\begin{aligned}
\left(v_{1} e_{1}+v_{2} e_{1}\right) e_{2}^{2} & =\left(v_{1} e_{1}+v_{2} e_{1}\right) e_{2} \\
& =v_{2} e_{1} \beta e_{2} .
\end{aligned}
$$

Since $e_{2}$ is central $v_{2} e_{1} \beta e_{2}=v_{2} e_{1} e_{2} \beta=0$. Thus $\left(v_{1} e_{1}+v_{2} e_{1}\right) e_{2}=0$ and $v_{1} e_{1}+v_{2} e_{1}$ is in $U$. It follows that $U$ is a submodule of $V$. Similarly $W$ is a submodule of $V$. If $v$ is in $V$, then $v=v e_{1}+v e_{2}$ and $V=U+W$. Also, if $v_{1} e_{1}=v_{2} e_{2}$ is in $U \cap W$ where $v_{1}$ and $v_{2}$ are in $V$, then $v_{1} e_{1}=v_{2} e_{2} e_{1}=\{0\}$. Hence $V=U \oplus W$. Now $A=e_{1} N$ and $W=V e_{2}$. Since $e_{2} e_{1}=0, A \leqq$ $(0: W)$. Also $B \leqq(O: U)$ and $(0: U)+(0: W)=N$. If $\alpha$ is in $(0: U) \cap(0: W)$, then since the sum $U+W$ is direct, $V \boldsymbol{\alpha}=\{0\}$. Since $V$ is faithful $(0: U) \cap(0: W)=\{0\}$. Thus $(0: U) \oplus(0: W)=N$ and, since $A \leqq(0: W)$ and $B \leqq(0: U)$, it follows that $A=(0: W)$ and $B=(0: U)$. The theorem is now proved.

\section{Finitely generated tame $N$-groups}

Let $V$ be a tame $N$-group. A submodule $U$ of $V$ is finitely generated if there exists $v_{i}, i=1, \ldots, k$, in $V$ such that

$$
U=\sum_{i=1}^{k} v_{i} N
$$


A standard result of ring theory (see (2, p. 96)) states that if $N$ is a ring with maximal condition on right ideals and $V$ a finitely generated $N$-module (in the ring sense), then a submodule, of $V$ is finitely generated. We shall show that this holds for tame $N$-groups.

A tame $N$-group $V$ will be called Noetherian if the submodules of $V$ satisfy the maximal condition.

The following proposition is the analogue of the corresponding ring result.

Proposition 3.1. A tame N-group $V$ is Noetherian if, and only if, every submodule of $V$ is finitely generated.

If $S$ is a set of subgroups of a group, then an element of $S$ will be called an $S$-subgroup. The next lemma, a lattice theory result, will be used again in Section five.

Lemma 3.2. Let $V$ be a group and $V_{i}, i=0, \ldots, k$, a properly ascending finite sequence of normal subgroups of $V$, such that $V_{0}=\{0\}$ and $V_{k}=V$. Let $S$ be a set of subgroups of $V$ such that $H \cap W$ and $H+W$ are in $S$ for all $H$ in $S$ and $W$ in $\left\{V_{i}: i=0, \ldots, k-1\right\}$. If the $S$-subgroups of $V$ between $V_{i}$ and $V_{i+1}$ satisfy minimal (maximal) condition for $i=0, \ldots$, $k-1$, then the elements of $S$ satisfy minimal (maximal) condition.

Proof. We shall prove the result for minimal condition as the proof for maximal condition is entirely similar.

For $k=1$ the result is trivial. Assume that the $S$-subgroups of $V$ between $\{0\}$ and $V_{k-1}$ satisfy minimal condition, and let

$$
H_{1} \geqq H_{2} \geqq \ldots
$$

be a descending chain of $S$-subgroups of $V$. By the assumptions there exists a positive integer $m$ such that $H_{m} \cap V_{k-1}=H_{m+n} \cap V_{k-1}$ and $H_{m}+V_{k-1}=H_{m+n}+V_{k-1}$ for $n=$ $0,1,2, \ldots$. Since $H_{m} \geqq H_{m+n}$, it follows by the "modular law" that $H_{m}=H_{m+n}$ for $n=0,1,2, \ldots$ The proof is now complete.

Proposition 3.3. Let $N$ be a near-ring with maximal condition on right ideals and $V a$ faithful tame $N$-group. If there exists $v$ in $V$ such that $v N=V$, then $V$ is Noetherian.

Proof. The obvious $N$-homomorphism of $N$ onto $v N$ establishes a one to one correspondence between the submodules of $v N$ and the right ideals of $N$ containing $(0: v)$. The proposition follows.

Theorem 3.4. Let $N$ be a near-ring with maximal condition on right ideals and $V a$ tame $N$-group. If $V$ is finitely generated, then any submodule of $V$ is finitely generated.

Proof. We have $V$ is a finite sum $\sum_{i=1}^{k} v_{i} N$ where $v_{i}, i=1, \ldots k$, are in $V$. The result will follow by 3.1 if we show that $V$ is Noetherian. We proceed by induction. The case $k=1$ follows from 3.3 Let $U=v_{2} N+\ldots+v_{k} N$. The submodule $U$ of $V$ may be assumed to be Noetherian by 3.1. Now $V / U$ is $N$-isomorphic to $v_{1} N /\left(v_{1} N\right) \cap U$ and $V / U$ is Noetherian by 3.3. From the isomorphism theorems it follows that the submodules of $V$ between $U$ and $V$ satisfy maximal condition. By $3.2 \mathrm{~V}$ is Noetherian and the theorem is proved. 


\section{Tame near-rings with minimal condition (the radical)}

Let $V$ be an $N$-group (assumed unitary). We shall call $V$ minimal if it is of type 2 (see (6, 3.5, p. 77)). This additional terminology is valuable for the case of tame $N$-groups. Indeed the only radical that will concern us here is $J_{2}(N)(\operatorname{see}(6,5.1, p .136))$, which we denote by $J(N)$.

If $N$ is a near-ring and $V$ an $N$-group, then an $N$-group $U / W$ will be called a factor of $V$ if $U \geqq W$ are submodules of $V$. The factor $U / W$ will be called minimal if $U>W$ and there exist no submodules of $V$ lying properly between $W$ and $U$. Thus $R_{1} / R_{2}$ is a minimal factor of a near-ring $N$, if $R_{1}>R_{2}$ are right ideals of $N$ and there exist no right ideals of $N$ lying properly between $R_{2}$ and $R_{1}$.

We aim to show that for a tame near-ring $N$ with minimal condition, $J(N)$ is nilpotent. First we show that a minimal factor of $N$ is a minimal $N$-group.

Proposition 4.1. If $N$ is a near-ring, $V$ a tame $N$-group, $M$ a right $N$-subgroup of $N$ and $v$ an element of $V$, then $M+(0: v)$ is a right ideal of $N$.

Proof. Let $\delta$ be the natural $N$-homomorphism of $N$ onto $v N$. Since $v M$ is a submodule of $v N$, the inverse image $M+(0: v)$, of $v M$ under $\delta$ is a right ideal of $N$.

Lemma 4.2. Let $N$ be a tame near-ring with minimal condition on right ideals. If $R_{1} / R_{2}$ is a minimal factor of $N$, then $R_{1} / R_{2}$ is a minimal $N$-group.

Proof. Out of all minimal factors of $N, N$-isomorphic to $R_{1} / R_{2}$, choose one $R / H$ such that $R$ is minimal. We show that $R / H$ is a minimal $N$-group. If $K<R$ is a right ideal of $N$, then $K \leqq H$, otherwise $K+H=R$ and $R / H$ is $N$-isomorphic to the minimal factor $K / K \cap H$. Let $M$ be a right $N$-subgroup of $N$ such that $H<M<R$ and let $V$ be a faithful tame $N$-group. Since the intersection $\cap(0: u)$ over all $u$ in $V$ is zero, there exists $v$ in $V$ such that $(0: v) \cap R<R$ and therefore $(0: v) \cap R \leqq H$. By $4.1 M+(0: v)$ is a right ideal of $N$. Thus

$$
R \cap[M+(0: v)]=M+(0: v) \cap R
$$

is a right ideal of $N$. But $(0: v) \cap R \leqq H<M$ and we see that $M$ is a right ideal of $N$. This contradiction completes the proof.

Theorem 4.3. If $N$ is a tame near-ring with minimal condition on right ideals, then $J(N)$ is nilpotent.

Proof. Suppose $J(N)$ is not nilpotent and $H \leqq J(N)$ is a right ideal of $N$ minimal for being non-nilpotent. If $R\left(H^{2}\right)<H$, then $H^{2}$ is nilpotent and so is $H$. Thus $R\left(H^{2}\right)=H$. We have $\{0\} H=\{0\}$ and by Zorn's Lemma we may find a right ideal $K_{1}$ of $N$ maximal for the property that $K_{1} H=\{0\}$. Since $N$ has an identity we may assume that $K_{1} \neq N$. By minimal condition there exists a right ideal $K_{2}>K_{1}$ with $K_{2} / K_{1}$ a minimal factor of $N$. By 4.2, $K_{2} / K_{1}$ is a minimal $N$-group. Since $H \leqq J(N),\left[K_{2} / K_{1}\right] H=\{0\}$ and $K_{2} H \subseteq K_{1}$. Thus $K_{2} H^{2} \leqq K_{1} H=\{0\}$. Hence $H^{2} \subseteq\left(0: K_{2}\right), H=R\left(H^{2}\right) \leqq\left(0: K_{2}\right)$ and it follows that $K_{2} H=$ $\{0\}$. This contradiction to the maximality of $K_{1}$ establishes the theorem. 


\section{Chain conditions on tame near-rings}

In this section it is proved that for tame near-rings minimal condition on right ideals implies minimal and maximal condition on right $N$-subgroups. This proof will be accomplished in a sequence of propositions and lemmas. We need certain preliminaries.

If $V$ is an $N$-group then a submodule $W$ of $V$ will be called absolutely reducible in $V$, if it is a direct sum of submodules of $V$ which are minimal $N$-groups.

The following result may be proved in the same way as for rings (see (2, p. 87)).

Proposition 5.1. If $N$ is a near-ring, $V$ an $N$-group and $W$ a submodule of $V$, then $W$ is absolutely reducible in $V$ if, and only if, $W$ is a sum of submodules of $V$ which are minimal N-groups.

If a submodule $W$ of an $N$-group $V$ is absolutely reducible in $V$, then $W J(N)=\{0\}$ (see $(6,2.29$, p. 49)). Furthermore, if $V$ has minimal condition on submodules, then $W$ is a finite direct sum of submodules which are minimal $N$-groups (see (2, p. 88)).

If $N$ is a non-zero tame near-ring with minimal condition on right ideals, then $\operatorname{soc} N$ will denote the sum of all minimal right ideals of $N$. By 4.2 and $5.1 \operatorname{soc} N$ is absolutely reducible in $N$. The above comment tells us that this direct sum is finite.

The following proposition can be deduced from (6, 5.32, p. 146).

Proposition 5.2. If $N$ is a near-ring with minimal condition on right ideals and $T$ is an ideal of $N$, then $J(N / T)=(J(N)+T) / T$.

Clearly for a tame near-ring $N$ with minimal condition, $\mathrm{RJ}(\mathrm{N})=\{0\}$ for all right ideals $R \leqq \operatorname{soc} N$. We now establish the converse.

Lemma 5.3. Let $N$ be a tame near-ring with minimal condition on right ideals and $R a$ right ideal of $N$ such that $R J(N)=\{0\}$. It follows that $R \leqq \operatorname{soc} N$.

Proof. Assume $R \equiv \operatorname{soc} N$ and let $H \leqq R$ be a right ideal of $N$ minimal for the property that $H \not \operatorname{soc} N$. Set $K=(\operatorname{soc} N) \cap H$. Clearly $\dot{K}<H$ and $K$ contains every right ideal of $N$ properly contained in $H$. By $(6,5.32$, p. 146) and 5.2

$$
N / J(N)=R_{1} \oplus \ldots \oplus R_{k}
$$

where the $R_{i}, i=1, \ldots, k$, are minimal right ideals and minimal $N / J(N)$-groups. Thus the $R_{i}$ are minimal $N$-groups. Let $\rho$ be in $H$ but not in $K$. Since $H$ is a unitary $N / J(N)$-group one of the $\rho R_{i}, i=1, \ldots, k$, say $\rho R_{j}$, must be such that $\rho R_{j} \not K$. Set $\rho R_{j}=M$. Since $R_{j}$ is a minimal $N$-group so is $M$. Hence $M \cap K=\{0\}$. Also, since $M \leqq H, M+K \leqq H$ and $M+K>K$. Now $K$ is maximal in $H$ and, by $4.2, M+K=H$.

If $K=\{0\}$, then $H=M$ and $H$ is a minimal right ideal. Thus we may assume that $K \neq\{0\}$. Let $V$ be a faithful tame $N$-group. There exists $v$ in $V$ such that $(0: v) \cap K<K$. Now either $(0: v) \cap H \geqq K$ or $(0: v) \cap H<K$. If $(0: v) \cap H \geqq K$, then $(0: v) \cap K=K$. Hence $(0: v) \cap H<K$. By $4.1, M+(0: v)$ is a right ideal of $N$ and thus

$$
H \cap[M+(0: v)]=M+(0: v) \cap H \quad\left(=K_{1} \text { say }\right)
$$

is a right ideal of $N$. Since $(0: v) \cap H<K, K_{1}<H$ and $K_{1} \leqq K$. Thus $M \leqq K$ and we have a contradiction that completes the proof. 
Lemma 5.4. Let $N$ be a tame near-ring with minimal condition on right ideals. If $S$ is a non-empty subset of $N$ such that $S J(N)=\{0\}$, then $S \subseteq \operatorname{soc} N$.

Proof. Let $\alpha$ be in $S$. We have $\alpha J(N)=\{0\}$. Since $N J(N) \subseteq J(N), \alpha N J(N)=\{0\}$. By 1.3, $R(\alpha N) J(N)=\{0\}$ and by 5.3, $R(\alpha N) \leqq \operatorname{soc} N$. Since $N$ has an identity $\alpha$ is in $R(\alpha N)$ and therefore in soc $N$. The lemma now follows.

Lemma 5.5. If $N$ is a tame near-ring with minimal condition on right ideals, then $\operatorname{soc} N$ is an ideal of $N$ and $N / \operatorname{soc} N$ is tame.

Proof. Let $V$ be a faithful tame $N$-group. If it is shown that there exists a submodule $U$ of $V$ such that $(U: V)=\operatorname{soc} N$, then it will follow that $\operatorname{soc} N$ is an ideal of $N$ and $N / \operatorname{soc} N$ will have $V / U$ as a faithful tame $N / \operatorname{soc} N$-group. We have soc $N$ is a finite direct sum $R_{1} \oplus R_{2} \oplus \ldots \oplus R_{k}$ of minimal right ideals which are minimal $N$-groups. Set

$$
U=\sum \sum v R_{i}
$$

where the sum is over all $i$ in $\{1, \ldots, k\}$ and $v$ in $V$. We have for each $i$ and $v$ that $v R_{i}=\{0\}$ or $v R_{i}$ is $N$-isomorphic to $R_{i}$. Since $V \operatorname{soc} N \neq\{0\}, U \neq\{0\}$ and it follows from 5.1 that $U$ is absolutely reducible in $V$. Obviously soc $N \leqq(U: V)$. Also $V(U: V) \subseteq U$ and, by the absolute reducibility of $U$

$$
V(U: V) J(N) \subseteq U J(N)=\{0\} .
$$

Since $V$ is faithful $(U: V) J(N)=\{0\}$. By $5.3,(U: V) \leqq \operatorname{soc} N$ and the lemma follows.

Our next lemma is the main step in proving that minimal condition on right ideals implies, for tame near-rings, maximal and minimal condition on right $N$-subgroups.

Lemma 5.6. If $N$ is a tame near-ring with minimal condition on right ideals, then there exists a positive integer $k$ and right ideals

$$
\{0\}=R_{0}<R_{1}<R_{2}<\ldots<R_{k}=N
$$

such that $R_{i+1} / R_{i}, i=0, \ldots, k-1$, are minimal factors of $N$.

Proof. By 4.3, $J(N)^{m}=\{0\}$ for some positive integer $m$. We assume $m$ is minimal and proceed by induction. If $m=1, J(M)=\{0\}$ and by $(6,5.32$, p. 146), $N$ is a finite direct sum

$$
H_{1} \oplus \ldots \oplus H_{n}
$$

of minimal right ideals of $N$. With $k=n, R_{0}=\{0\}$ and $R_{i}=H_{1} \oplus \ldots \oplus H_{i}, i=1, \ldots, k$, the lemma follows in this case.

Assume $m>1$. Now $J(N)^{m-1} J(N)=\{0\}$ and by $5.4, J(N)^{m-1} \cong \operatorname{soc} N$. Also by 5.2

and, since

$$
J(N / \operatorname{soc} N)=(J(N)+\operatorname{soc} N) / \operatorname{soc} N
$$

we conclude that

$$
(J(N)+\operatorname{soc} N)^{m-1} \subseteq J(N)^{m-1}+\operatorname{soc} N \cong \operatorname{soc} N,
$$

$$
[J(N / \operatorname{soc} N)]^{m-1}=\{0\} .
$$


Since, by $5.5, N / \operatorname{soc} N$ is tame it follows from the induction assumption that there exists a positive integer $s$ and right ideals $K_{i}, i=0, \ldots, s$, of $N / \operatorname{soc} N$ such that

$$
\{0\}=K_{0}<K_{1}<\ldots<K_{s}=N / \operatorname{soc} N
$$

where $K_{i+1} / K_{i}, i=0, \ldots, s-1$, are minimal factors of $N / \operatorname{soc} N$. Now there exists right ideals $K_{i}^{\prime}, i=0, \ldots, s$, of $N$ containing $\operatorname{soc} N$ and such that $K_{i}^{\prime} / \operatorname{soc} N=K_{i}$. Also $\operatorname{soc} N$ is a finite direct sum $B_{1} \oplus \ldots \oplus B_{r}$ of minimal right ideals of $N$. Set $K=r+s, R_{0}=\{0\}, R_{i}=B_{1} \oplus \ldots \oplus B_{i}$ for $i \leqq r$ and $R_{i} \doteq K_{i-r}^{\prime}$ for $i<r$ and $i \leqq k$. The fact that the factors $R_{i+1} / R_{i}, i=0, \ldots, k-1$. are minimal follows from the isomorphism theorems. The lemma is now proved.

Theorems 5.7. If $N$ is a tame near-ring with minimal condition on right ideals, then $N$ has minimal and maximal condition on right $N$-subgroups.

Proof. By 5.6, $N$ has a finite sequence

$$
\{0\}=R_{0}<R_{1}<R_{2}<\ldots<R_{k}=N
$$

of right ideals of $N$ such that $R_{i+1} / R_{i}, i=0, \ldots, k-1$, are minimal factors of $N$. Let $S$ be the set of all right $N$-subgroups of $N$. By 4.2 , there are no elements of $S$ lying properly between $R_{i+1}$ and $R_{i}$ for $i=0, \ldots, k-1$. It follows from 3.2 that the elements of $S$ satisfy both chain conditions. The theorem is therefore proved.

\section{Compatible and 2-tame $N$-groups}

We return to the example of a tame $N$-group given in the introduction (viz. a near-ring generated by a semi-group $S$ of endomorphisms of $V$ where $S$ contains Inn $(V)$, the inner automorphisms of $V$ ). As indicated there, such an $N$-group has a much stronger property than simply being tame.

Suppose $\mu$ or $-\mu$ is in $S$ and $v$ in $V$. We have $(v+w) \mu-v \mu$ is equal to either $v \mu+w \mu-v \mu$ or $w \mu$ for all $w$ in $V$. Since $\operatorname{Inn}(V) \subseteq S$, it follows that there exists $\beta$ in $N$ such that

$$
(v+w) \mu-v \mu=w \beta
$$

for all $w$ in $V$. Now suppose that for an $\alpha$ in $N$, there exists $\gamma$ in $N$ such that

$$
(v+w) \alpha-v \alpha=w \gamma
$$

for all $w$ in $V$ and take $\lambda=\alpha+\mu$. Computing we see that

$$
\begin{aligned}
(v+w) \lambda-v \lambda & =(v+w)(\alpha+\mu)-v(\alpha+\mu) \\
& =(v+w) \alpha+(v+w) \mu-v \mu-v \alpha \\
& =(v+w) \alpha-v \alpha+v \alpha+w \beta-v \alpha \\
& =w[\gamma+\beta \kappa]
\end{aligned}
$$

where $\kappa$ is the inner automorphism of $V$ induced by $v \alpha$. It follows therefore by induction that given any $\alpha$ in $N$ there exists $\gamma$ in $N$ such that

$$
(v+w) \alpha-v \alpha=w \gamma
$$

for all $w$ in $V$. This is a stronger version of 2.1 . We are ready for a definition. 
Let $N$ be a near-ring. An $N$-group $V$ will be called compatible if, given $v$ in $V$ and $\alpha$ in $N$, there exists $\beta$ in $N$ such that

$$
(v+w) \alpha-v \alpha=w \beta
$$

for all $w$ in $V$.

Example. Let $V$ be an $\Omega$-group belonging to some variety $\mathscr{V}$. Let $V(x, \mathscr{V})$ be the polynomials in $x$ over $V$ (see $(6$, p. 215) or $(4$, pp. 12-13)). Now $V(x, \mathscr{V})$ is a near-ring (not necessarily zero-symmetric) under substitution (for $x)$ and pointwise addition. If $(x) p$ is in $V(x, \mathscr{V})$, then the maps of $V$ into $V$ of the form $u \rightarrow u p(u \in V)$ form a near-ring (this near-ring, the near-ring of polynomial maps (4), is not necessarily zero-symmetric). We denote the zero-symmetric part of this near-ring by $P_{0}(V)$. We claim that $V$ is a compatible $P_{0}(V)$-group. Indeed, if $\alpha$ is in $P_{0}(V)$ and $v$ in $V$, then there exists $(x) p$ in $V(x, \mathscr{V})$ where $\alpha$ is the map $u \rightarrow u p(u \in V)$. Clearly $(x) q=(v+x) p-v p$ is in $V(x, \mathscr{V})$ and thus with $\beta$ the map $u \rightarrow u q(u \in V)$, it follows that

$$
(v+u) \alpha-v \alpha=u \beta
$$

for all $u$ in $V$. This example illustrates the importance of compatible $N$-groups.

The notion of a 2-tame $N$-group will now be introduced. Let $V$ be an $N$-group. Suppose that for each $v$ in $V$ and $\alpha$ in $N$ there exists $\beta$ in $N$ such that

$$
\left(v+w_{i}\right) \alpha-v \alpha=w_{i} \beta
$$

for any two elements $w_{i}, i=1,2$, of $V$. In this case we call $V 2$-tame.

Clearly being 2-tame is stronger than being tame and likely to be weaker than being compatible. It is also clear from the definition that one may define an $n$-tame $N$-group where $n$ is any cardinal.

If a near-ring $N$ has a faithful 2-tame (compatible) $N$-group, then we call $N$ 2-tame (compatible).

\section{7. $\mathbf{N}$-endomorphisms of 2-tame $\mathbf{N}$-groups}

Although the $N$-endomorphisms of an $N$-group $V$ need not form a near-ring the situation is simplified in the case that $V$ is 2-tame by the fact that if $\delta$ is an $N$ endomorphism then so is $1-\delta$.

The following proposition is basic.

Proposition 7.1. If $V$ is a 2-tame $N$-group and $\mu$ an $N$-endomorphism of $V$, then

$$
(v+w \mu) \alpha-v \alpha=(v \mu+w \mu) \alpha-v \mu \alpha
$$

for all $v$ and $w$ in $V$ and $\alpha$ in $N$.

Proof. Since $V$ is 2 -tame, there exists $\beta$ in $N$ such that

$$
\begin{aligned}
& (v+w) \alpha-v \alpha=w \beta ; \quad \text { and } \\
& (v+w \mu) \alpha-v \alpha=w \mu \beta .
\end{aligned}
$$


Now

$$
\begin{aligned}
w \mu \beta & =w \beta \mu \\
& =[(v+w) \alpha-v \alpha] \mu \\
& =(v \mu+w \mu) \alpha-(v \mu \alpha)
\end{aligned}
$$

and the proposition follows.

Let $V$ be an $N$-group and $\delta$ an $N$-endomorphism of $V$. By $1-\delta$ we mean the map of $V$ into $V$ taking $v$ in $V$ to $v-v \delta$.

Proposition 7.2. If $V$ is a 2-tame $N$-group and $\delta$ an $N$-endomorphism of $V$, then $u(1-\delta)$ commutes with $w \delta$ for all $u$ and $w$ in $V$.

Proof. In 7.1 take $\alpha=1, \mu=\delta$ and $v=-u$. It follows that

$$
-u+w \delta+u=-u \delta+w \delta+u \delta
$$

for all $w$ in $V$ (i.e. $-u(1-\delta)+w \delta+u(1-\delta)=w \delta$ ). The proposition follows.

Theorem 7.3. If $V$ is a 2-tame $N$-group and $\delta$ an $N$-endomorphism of $V$, then $1-\delta$ is an $N$-endomorphism.

Proof. Let $v_{1}$ and $v_{2}$ be in $V$. We have

$$
\left(v_{1}+v_{2}\right)(1-\delta)=v_{1}+v_{2}-v_{2} \delta-v_{1} \delta .
$$

Since by $7.2, v_{2}-v_{2} \delta$ commutes with $v_{1} \delta$, it follows that

$$
\begin{aligned}
\left(v_{1}+v_{2}\right)(1-\delta) & =v_{1}-v_{1} \delta+v_{2}-v_{2} \delta \\
& =v_{1}(1-\delta)+v_{2}(1-\delta) .
\end{aligned}
$$

Thus $1-\delta$ is a group homomorphism of $V$ into $V$. Now take $\mu=\delta$ and $w=-v$ in 7.1. We see that

$$
(v-v \delta) \alpha-v \alpha=-(v \delta \alpha)
$$

for all $v$ in $V$ and $\alpha$ in $N$. Thus

$$
v(1-\delta) \alpha=-(v \alpha \delta)+v \alpha .
$$

By 7.2, va $\delta$ commutes with $v \alpha-v \alpha \delta$ and

It follows that

$$
-(v \alpha \delta)+v \alpha=v \alpha-v \alpha \delta .
$$

$$
\begin{aligned}
v(1-\delta) \alpha & =v \alpha-v \alpha \delta \\
& =v \alpha(1-\delta)
\end{aligned}
$$

The theorem is now proved.

If $V$ is an $N$-group we denote the centre of $V$ by $Z(V)$. Theorem 7.3 has the following corollary.

Corollary. If $V$ is a 2-tame $N$-group and $\delta$ an $N$-endomorphism of $V$, then $V \delta$ and $V(1-\delta)$ are submodules of $V, V=V(1-\delta)+V \delta$ and $V \delta \cap V(1-\delta) \leqq Z(V)$. 
Proof. Since $1-\delta$ is an $N$-endomorphism, $V \delta$ and $V(1-\delta)$ are $N$-subgroups of $V$ and thus submodules. Now if $v$ is in $V, v=v-v \delta+v \delta$ and therefore $V(1-\delta)+V \delta=V$. By $7.2, V(1-\delta) \cap V \delta$ is in the centre of $V \delta$ and $V(1-\delta)$. Therefore $V \delta \cap V(1-\delta) \leqq Z(V)$ and the corollary follows.

An interesting special case of the above corollary is where $Z(V)=\{0\}$. In this case if $\delta$ is non-trivial, then neither $V(1-\delta)$ nor $V \delta$ is $\{0\}$ and $V=V(1-\delta) \oplus V \delta$. Thus in this case every $N$-endomorphism of $V$ induces a unique split of $V$ and a strong version of the Krull-Schmitt theorem holds.

We now look at the case where $Z(V) \neq\{0\}$. A non-zero $N$-group $V$ will be called indecomposible if $V$ is not the direct sum of two non-zero submodules. If $V$ is a 2-tame $N$-group, the proof of Lemma 14.4 p. 82 of (2) goes through without change. From this the following theorem is easily deduced.

Theorem 7.4. Let $N$ be a near-ring with a 2-tame $N$-group $V$ where $V$ has maximal and minimal condition on submodules. If

$$
V=U_{1} \oplus \ldots \oplus U_{r}=W_{1} \oplus \ldots \oplus W_{s}
$$

where the $U_{i}, i=1, \ldots, r$, and $W_{i}, i=1, \ldots, s$, are indecomposible, then $r=s$ and there exists $a$ permutation $p$ of $1, \ldots, r$ such that $U_{i}$ is $N$-isomorphic to $W_{(i) p} i=1, \ldots, r$.

\section{Primitive 2-tame near-rings}

We call $N$ a primitive 2-tame near-ring, if it has a 2-tame $N$-group which is minimal and faithful.

Let us denote the near-ring of all zero-fixing maps of a group $V$ by $M_{0}(V)$.

It is the purpose of this section to look more closely at primitive 2-tame near-rings. Indeed in the case where $N$ has minimal condition these near-rings are either of the form $M_{0}(V)$ (where $V$ is a finite group) or a complete matrix ring over a division ring.

Lemma 8.1. Let $N$ be a near-ring, $V$ a minimal $N$-group which is 2-tame, Aut $_{N} V$ the group of all $N$-automorphisms of $V$ and 0 the zero $N$-endomorphism of $V$. If $\operatorname{Aut}_{N} V \neq\{1\}$ then $\mathrm{Aut}_{N} V \cup\{0\}$ is a division ring under composition and pointwise addition.

Proof. Since $V$ is minimal, any non-trivial $N$-endomorphism of $V$ is in $A_{u t} V$. Let $\delta \neq 1$ be in $\mathrm{Aut}_{N} V$. By 7.3, $1-\delta$ and $1-\delta^{-1}$ are in Aut $_{N} V$. But $\delta\left(1-\delta^{-1}\right)=\delta-1$. Thus $\delta-1$ is in $\operatorname{Aut}_{N} V$. Hence $(1-\delta)(\delta-1)^{-1}$ is in Aut $_{N} V$. Now

$$
\begin{aligned}
v(1-\delta)(\delta-1)^{-1} & =-v(\delta-1)(\delta-1)^{-1} \\
& =-v
\end{aligned}
$$

for all $v$ in $V$, and the map $-1: v \rightarrow-v$ is in Aut $_{N} V$. It follows that $V$ is abelian.

We shall show that Aut $_{N} V \cup\{0\}$ is an abelian group under addition. Clearly 0 is an additive identity of Aut $_{N} V \cup\{0\}$ and, if $\mu$ is in Aut $\left.N V \cup 0\right\}$, then $-\mu=(-1) \mu$ is an additive inverse of $\mu$. Suppose $\delta_{1}$ and $\delta_{2}$ are in Aut $V \cup\{0\}$. If either $\delta_{1}$ or $\delta_{2}$ is zero then it is certainly true that $\delta_{1}-\delta_{2}$ is in Aut ${ }_{N} V \cup\{0\}$. If $\delta_{1}=(-1) \delta_{2}=-\delta_{2}$, then $\delta_{1}+\delta_{2}=0$, and if $\delta_{1} \neq(-1) \delta_{2}$ then $1-\delta_{1}^{-1}(-1) \delta_{2}$ is in Aut $_{N} V$ by 7.3. In this case

$$
\delta_{1}\left(1-\delta_{1}^{-1}(-1) \delta_{2}\right)=\delta_{1}-(-1) \delta_{2}=\delta_{1}+\delta_{2}
$$


is in Aut $_{N} V$. Hence, under addition, Aut $_{N} V \cup\{0\}$ is a group. This group is abelian since $V$ is abelian. The left distributive law clearly holds and since the elements of Aut $_{N} V \cup\{0\}$ are distributive in $M_{0}(V)$ the right distributive law holds. Also a non-zero element of Aut $_{N} V \cup\{0\}$ has a multiplicative inverse and the lemma is proved.

Lemma 8.2. If $N$ is a near-field with a minimal 2-tame $N$-group $V$, then $N$ is a division ring.

Proof. By $(6,8.3$, p. 237) $N$ has no proper right $N$-subgroups $(0: V)=\{0\}$, and $N$ is faithful on $V$. Clearly if $V$ has only one non-zero element, then $N$ is the field of order two. Now if $u$ and $v$ are two non-zero elements of $V$, then $(0: u)=0=(0: v)$ and there exists an $N$-isomorphism $\mu$ of $u N(=V)$ onto $v N(=V)$ such that $u \mu=v$. Now, by $8.1,1+\mu$ is an $N$-endomorphism of $V$. If $\alpha$ is in $N$, then

$$
\begin{aligned}
(u+v) \alpha & =(u+u \mu) \alpha \\
& =u \alpha(1+\mu) \\
& =u \alpha+u \mu \alpha \\
& =u \alpha+v \alpha
\end{aligned}
$$

and the elements of $N$ distributive over $V$. Now $V \neq\{0\}$ and, since $V$ is a unitary $N$-group, $(0: V)=\{0\}$. The elements of $N$ are distributive in $M_{0}(V)$ and the lemma follows.

Before we state our main result on primitive 2-tame near-rings we need a proposition.

A submodule $U$ of an $N$-group $V$ will be called abelian if addition in $U^{+}$is commutative and elements of $N$ distribute over $U$ (i.e. if $N /(0: U)(=A)$ is a ring and $U$ is an $A$-module in the ring sense).

Proposition 8.3. If an $N$-group $V$ is a direct sum $V_{1} \oplus V_{2}$ of two minimal $N$-groups and if $V_{1}$ is non-abelian, then $V_{1}$ and $V_{2}$ are the only proper submodules of $V$.

Proof. If $W$ is a proper submodule of $V$ and $W \neq V_{i}, i=1,2$, then by $(6,2.23$, p. 48)

is abelian. But

$$
\left(V_{1}+W\right) \cap\left(V_{2}+W\right) / W
$$

$$
V_{1}+W=V_{2}+W=V
$$

and, since $V / W$ is $N$-isomorphic to

$$
V_{1} / V_{1} \cap W=V_{1} /\{0\},
$$

$V_{1}$ is abelian. This contradiction establishes the proposition.

If $N$ is a near-ring and $V$ a faithful minimal $N$-group we may regard $N$ as a subnear-ring of $M_{0}(V)$. We say $N$ is dense in $M_{0}(V)$, if it is dense with respect to the finite topology (see $(6,4.26$, p. 111)).

Theorem 8.4. Let $N$ be a near-ring with a faithful minimal $N$-group $V$. The $N$-group $V$ is 2-tame if, and only if, $N$ is either dense in $M_{0}(V)$ or $N$ is a ring.

Proof. Suppose $N$ is a ring. Since for $v \neq 0$ in $V, v N=V$, it is evident that $V$ is a ring 
module. Thus if $v$ and $w$ are in $V$ and $\alpha$ is in $N$, then $(v+w) \alpha-v \alpha=w \alpha$. Clearly $V$ is 2-tame.

Suppose $N$ is dense in $M_{0}(V)$. It follows that if $w_{1}$ and $w_{2}$ are any two distinct elements of $V$, then there exists $\beta$ in $V$ such that $w_{1} \beta=v_{1}$ and $w_{2} \beta=v_{2}$ for any two elements $v_{1}$ and $v_{2}$ of $V$. Let $v$ be in $V$ and $\alpha$ in $N$ and take

$$
v_{i}=\left(v+w_{i}\right) \alpha-v \alpha, \quad i=1,2 .
$$

The fact that $V$ is 2-tame follows from the existence of $\beta$.

Now suppose that $V$ is 2 -tame and that $N$ is not a ring. The result will follow from (6, 4.54 , p. 129) if we can show that an $N$-automorphism $\mu$ of $V$ must in fact be the identity.

Let $v$ be a non-zero element of $V$. If for all non-zero elements $u$ of $V,(0: v)=(0: u)$, then $(0: v)=\cap(0: u)$ where $u$ ranges over all elements of $V$. In this case $(0: v)=0$, since $(0: V)=\{0\}$. Therefore $v N(=V)$ is $N$-isomorphic to $N$ and $N$ has no proper right $N$-subgroups. By $(6,8.3$, p. 237) $N$ is a near-field and by $8.2, N$ is a division ring.

Thus we may assume that there exists a non-zero element $u$ in $V$ such that $(0: u) \neq(0: v)$. If $\alpha$ is in $(0: v)$, then $v \alpha=v \mu \alpha=0$ and, by 7.1 it follows that $(v+w \mu) \alpha=$ $(v \mu+w \mu) \alpha$ for all $w$ in $V$. Thus

$$
\left(v+w_{1}\right) \alpha=\left(v \mu+w_{1}\right) \alpha
$$

for all $w_{1}$ in $V$ and $\alpha$ in $(0: v)$. Now take $w_{1}$ such that $v+w_{1}=u$ and set $x=v \mu+w_{1}$. Since $v \neq 0,(0: v)$ is, by $(6$, p. 103), a maximal right ideal of $\mathrm{N}$. Also $(0: u) \neq(0: v)$. Thus $(0: u)+(0: v)=N$ and $1=e_{1}+e_{2}$ where $e_{1}$ is in $(0: u)$ and $e_{2}$ is in $(0: v)$. Now

$$
u=u e_{1}+u e_{2}=u e_{2}=x e_{2}
$$

by (1). Since $x e_{2} \neq 0, x \neq 0$. We shall show that $(0: u) \cap(0: v) \leqq(0: x)$. If $\gamma$ is in $(0: u) \cap(0: v)$ then, since $V$ is 2-tame, there exists $\lambda$ in $N$ such that

and

$$
\left(v+w_{1}\right) \gamma-v \gamma=w_{1} \lambda
$$

$$
\left(v+w_{1} \mu^{-1}\right) \gamma-v \gamma=w_{1} \mu^{-1} \lambda .
$$

Now $v+w_{1}=u$ and, since $\gamma$ is in $(0: u) \cap(0: v)$, it follows that $w_{1} \lambda=0$. Therefore $w_{1} \mu^{-1} \lambda=w_{1} \lambda \mu^{-1}=0$. Thus

$$
\left(v+w_{1} \mu^{-1}\right) \gamma-v \gamma=0
$$

and, since $v \gamma=0$, we see that $\left(v+w_{1} \mu^{-1}\right) \gamma=0$. It now follows that

$$
\begin{aligned}
0 & =\left(v+w_{1} \mu^{-1}\right) \gamma \mu \\
& =\left(v \mu+w_{1}\right) \gamma \\
& =x \gamma .
\end{aligned}
$$

Hence $\gamma$ is in $(0: x)$ and $(0: u) \cap(0: v) \leqq(0: x)$.

Now $x \neq 0$ and $(0: x)$ is a maximal right ideal of $N$. By (2) $x e_{2} \neq 0$ and $(0: x) \neq(0: v)$. Thus $(0 ; x)+(0: v)=N$ and with $R=(0: u) \cap(0: v)$, it follows that

$$
N / R=(0: x) / R+(0: v) / R=(0: u) / R \oplus(0: v) / R .
$$

Now $(0: u) / R$ is $N$-isomorphic to $N /(0: v)$ which is $N$-isomorphic to $v N(=V)$. Similarly $(0: v) / R$ is $N$-isomorphic to $V$. Since $(0: x) / R$ is a proper submodule of $N / R$, it follows by 
8.3 that $(0: x) / R$ is equal to either $(0: u) / R$ or $(0: v) / R$. But since $(0: x) \neq(0: v)$, $(0: u) / R=(0: x) / R$ and $(0: u)=(0: x)$. Thus $x=x e_{2}$. By (2) $u=x$. Thus $v+w_{1}=v \mu+w_{1}$ and $v=v \mu$. This is true for any $v$ in $V$ and $\mu$ is therefore the identity. The proof is complete.

In Section 5 we saw that for tame near-rings minimal condition on right ideals implies maximal condition on right ideals. However, if 2-tame near-rings depart far enough from rings, minimal condition implies finiteness.

We shall call a near-ring $N$ ring-free if no non-zero homomorphic image of $N$ is a ring.

With the aid of 8.4 it is possible to deduce the following theorem.

Theorem 8.5. If $N$ is a 2-tame ring-free near-ring with minimal condition on right ideals then $N$ is finite.

This is proved by considering right ideals

$$
\{0\}=R_{0}<R_{1}<\ldots<R_{k}=N
$$

as in lemma 5.6 and, out of all minimal factors of $N, N$-isomorphic to $R_{i+1} / R_{i}$ ( $i$ in $\{0, \ldots, k-1\}$ ) choosing one $R / H$ with $R$ minimal. Then, as in the proof of 4.2 , one finds an element $v$ of a faithful 2-tame $N$-group $V$ such that $v R>v H$. The proof is completed by observing that the 2-tame $N$-group $v R / v H$ is $N$-isomorphic to $R_{i+1} / R_{i}$ from which it follows, by 8.4 and $(6,4.61$, p. 132$)$, that $R_{i+1} / R_{i}$ is finite.

\section{Centralisers of abelian submodules}

In this section we prove certain specialised results to be used in proving the final theorem in the next section.

Let $V$ be a group and $H_{1}$ and $H_{2}$ subsets of $V$. By $\left[H_{1}, H_{2}\right]$ we shall mean the subset of $V$ consisting of all $-h_{1}-h_{2}+h_{1}+h_{2}$ where $h_{i}$ is in $H_{i}, i=1,2$.

Let $U$ be a submodule of a tame $N$-group $V$, then $C_{V}(U)$ will denote the set of all elements $v$ in $V$ such that $[v N, U]=\{0\}$.

Proposition 9.1. If U is a submodule of a tame $N$-group $V$, then $C_{V}(U)$ is a submodule.

Proof. Since the set of all $v$ in $V$ such that $[v, U]=\{0\}$ forms a subgroup $H$ of $V$, $C_{V}(U)$ is simply the sum of all submodules of $V$ contained in $H$.

From now on we shall be dealing with the following situation.

(a) $N$ is a near-ring with minimal condition on right ideals;

(b) $V$ is a faithful compatible $N$-group;

(c) $U$ is an abelian minimal $N$-subgroup of $V$; and

(d) $(U: V) \nsubseteq(0: U)$.

Proposition 9.2. There exists $\gamma$ in $(U: V)$ such that $u \gamma=u$ for all $u$ in $U$.

Proof. Since $U$ is minimal and abelian and $N$ has minimal condition $N /(0: U)$ is a simple ring. Thus $(0: U)$ is a maximal ideal of $N$ and by (d), $(0: U)+(U: V)=N$. Let 
$1=\gamma_{1}+\gamma_{2}$ where $\gamma_{1}$ is in $(0: U)$ and $\gamma_{2}$ is in $(U: V)$. If we take $\gamma=\gamma_{2}$ the proposition follows.

Lemma 9.3. Let $v_{i}, i \in I$, be a system of coset representatives of $V / U$ in $V$. For each $k$ in I there exists $\xi_{k}$ in $N$ such that

(i) $\xi_{k} \equiv 1 \bmod (U: V)$;

(ii) $\left(v_{k}+u\right) \xi_{k}=\left(v_{k}\right) \xi_{k}$ for all $u$ in $U$; and

(iii) $u \xi_{k}=0$ for all $u$ in $U$.

Proof. Let $\gamma$ be as in 9.2. There exists $\alpha_{k}$ in $N$ such that

$$
\left(-v_{k}+v\right) \gamma-\left(-v_{k}\right) \gamma=v \alpha_{k}
$$

for all $v$ in $V$. Since $\gamma$ is in $(U: V)$, it follows that for all $v$ in $V, v \alpha_{k}$ is in $U$ and $\alpha_{k}$ is in $(U: V)$. Set $\rho_{k}=1-\alpha_{k}$. Clearly $\rho_{k} \equiv 1 \bmod (U: V)$. Now

$$
\begin{aligned}
\left(v_{k}+u\right) \rho_{k} & =v_{k}+u-\left(v_{k}+u\right) \alpha_{k} \\
& =v_{k}+u-\left[\left(-v_{k}+v_{k}+u\right) \gamma-\left(-v_{k}\right) \gamma\right] \\
& =v_{k}+u+\left(-v_{k}\right) \gamma-u \gamma
\end{aligned}
$$

for all $u$ in $U$. Since $\left(-v_{k}\right) \gamma$ is in $U, U$ is abelian and $u \gamma=u$ for all $u$ in $U$, it follows that

$$
\left(v_{k}+u\right) \rho_{k}=v_{k}+\left(-v_{k}\right) \gamma
$$

for all $u$ in $U$. Taking $u=0$ we see that $v_{k}+\left(-v_{k}\right) \gamma=v_{k} \rho_{k}$ and

$$
\left(v_{k}+u\right) \rho_{k}=v_{k} \rho_{k}
$$

for all $u$ in $U$. Suppose, without loss of generality, that 0 is in $I$ and $v_{0}$ represents the coset $U$. Take $\xi_{k}=\rho_{k} \rho_{0}$. Now $u \rho_{0}=v_{0} \rho_{0}$ for all $u$ in $U$ and since $0 \rho_{0}=0$, it follows that $u \rho_{0}=0$ for all $u$ in $U$. Thus $u \xi_{k}=u \rho_{k} \rho_{0}=0$ for all $u$ in $U$ since $u \rho_{k}$ is in $U$. We have shown (iii) holds. Also, since $\rho_{k} \equiv \rho_{0} \equiv 1 \bmod (U: V), \xi_{k} \equiv 1 \bmod (U: V)$ and $(i)$ holds. Finally

$$
\begin{aligned}
\left(v_{k}+u\right) \xi_{k} & =v_{k} \rho_{k} \rho_{0} \\
& =v_{k} \xi_{k}
\end{aligned}
$$

for all $\boldsymbol{u}$ in $U$ and (ii) holds. The lemma is proved.

Lemma 9.4. Let $v_{i}, i \in I$ be a system of coset representatives of $V / U$ in $V$. Let $S$ be the subset of I consisting of all $s$ in I such that $v_{s} \equiv 0 \bmod C_{V}(U)$. If $S$ is a proper subset of I and $k$ is in $I-S$, then there exists $A_{k}$ in $(U: V)$ such that

(i) $\left(v_{k}+u_{1}\right) \lambda_{k} \neq v_{k} \lambda_{k}$ for some $u_{1}$ in $U$; and

(ii) $u \lambda_{k}=0$ for all $u$ in $U$.

Proof. Let $\xi_{k}$ be as in 9.3. Set $\sigma_{k}=-\xi_{k}+1$. Clearly $\alpha_{k}$ is in $(U: V)$ and

$$
\begin{aligned}
\left(v_{k}+u\right) \alpha_{k} & =-v_{k} \xi_{k}+v_{k}+u \\
& =v_{k} \alpha_{k}+u
\end{aligned}
$$

for all $u$ in $U$. Since $v_{k}$ is not in $C_{v}(U)$, there exists $\eta$ in $N$ such that

$$
\left[v_{k} \eta, U\right] \neq 0 \text {. }
$$


Take $\lambda_{k}=\left[\eta, \alpha_{k}\right]$. Clearly $\lambda_{k}$ is in $(U: V)$. If $u$ is in $U$, then

$$
\begin{aligned}
u \lambda_{k} & =-u \eta-u \alpha_{k}+u \eta+u \alpha_{k} \\
& =0
\end{aligned}
$$

since $U$ is abelian. Thus (ii) holds. If $u$ is in $U$ then, since $U$ is a submodule of $V$,

$$
\left(v_{k}+u\right) \eta=u^{\prime}+\left(v_{k}\right) \eta
$$

where $u^{\prime}$ is in $U$. Thus

$$
\begin{aligned}
\left(v_{k}+u\right) \lambda_{k} & =-\left(v_{k}\right) \eta-u^{\prime}-\left(v_{k}+u\right) \alpha_{k}+u^{\prime}+v_{k} \eta+\left(v_{k}+u\right) \alpha_{k} \\
& =\left[v_{k} \eta,\left(v_{k}+u\right) \alpha_{k}\right],
\end{aligned}
$$

since $\left(v_{k}+u\right) \alpha_{k}$ is in $U$ and $U$ is abelian. From (1) and the fact that $v_{k} \alpha_{k}$ is in $U,\left(v_{k}+u\right) \alpha_{k}$ takes all values of $U$ as $u$ ranges over $U$. From (2) and (3)

$$
\left(v_{k}+u\right) \lambda_{k} \neq 0
$$

for some $u$ in $U$. If in (3) we take $u_{2}=-\left(v_{k}\right) \alpha_{k}$, then from (1), $\left(v_{k}+u_{2}\right) \alpha_{k}=0$ and

$$
\begin{aligned}
\left(v_{k}+u_{2}\right) \lambda_{k} & =\left[v_{k} \eta, 0\right] \\
& =0 .
\end{aligned}
$$

Now (i) follows as a consequence of (4) and (5). The lemma is proved.

Lemma 9.5. Let $v_{i}, i \in I$. be a system of coset representatives of $V / U$ in $V$. Let $S$ be the subset of I consisting of all $s$ in I such that $v_{s} \equiv 0 \bmod C_{V}(U)$.

If $S$ is a proper subset of $I$ and $k$ is in $I-S$, then there exists $\gamma_{k}$ in $(U: V)$ such that

(i) $\left(v_{k}+u\right) \gamma_{k}=v_{k} \gamma_{k}$ for all $u$ in $U$; and

(ii) $u_{1} \gamma_{k} \neq 0$ for some $u_{1}$ in $U$.

Proof. Let $v_{i}$ be the representative of the coset $-v_{k}+U$ of $U$. Let $\lambda_{i}$ be as in 9.4. Take $\gamma_{k}$ to be an element of $N$ such that

$$
\left(v_{i}+v\right) \lambda_{i}-v_{i} \lambda_{i}=v \gamma_{k}
$$

for all $v$ in $V$. Since $\lambda_{i}$ is in $(U: V)$ so is $\gamma_{k}$. Now

$$
\left(v_{i}+u_{1}\right) \lambda_{i} \neq v_{i} \lambda_{i}
$$

for some $u_{1}$ in $U$ and thus $u_{1} \gamma \neq 0$. Hence ( $\left.i i\right)$ holds. Also

$$
\left(v_{k}+u\right) \gamma_{k}=\left(v_{i}+v_{k}+u\right) \lambda_{i}-v_{i} \lambda_{i}
$$

for all $u$ in $U$. Since $v_{i}+v_{k}$ is in $U,\left(v_{i}+v_{k}+u\right) \lambda_{i}=0$ and

$$
\left(v_{k}+u\right) \gamma_{k}=-v_{i} \lambda_{i}
$$

for all $\boldsymbol{u}$ in $U$. On taking $u=0$ we see that $v_{k} \gamma_{k}=-v_{i} \lambda_{i}$. Thus

$$
\left(v_{k}+u\right) \gamma_{k}=v_{k} \gamma_{k}
$$

for all $u$ in $U$. Thus (i) follows and the lemma is proved. 
Lemma 9.6. Let $v_{i}, i \in I$, be a system of coset representatives of $V / U$ in $V$. Let $S$ be the subset of I consisting of all $s$ in $I$ such that $v_{s} \equiv 0 \bmod C_{V}(U)$. If $S$ is a proper subset of I and $k$ is in $I-S$, then there exists $\delta_{k}$ in $N$ such that

(i) $\delta_{\mathrm{k}} \equiv 1 \bmod (U: V)$;

(ii) $\left(v_{k}+u\right) \delta_{k} \equiv v_{k} \delta_{k}$ for all $u$ in $U$; and

(iii) $u \delta_{k}=u$ for all $u$ in $U$.

Proof. Let $\gamma_{k}$ be as in 9.5 and $\bar{\gamma}_{k}$ be the image of $\gamma_{k}$ in $N /(0: U)$. By (ii) of 9.5 , $u_{1} \bar{\gamma}_{k} \neq 0$ for some $u_{1}$ in $U$. Since $U$ is abelian, $N /(0: U)$ is a ring which is primitive on $U$ and has minimal condition. Thus $N /(0: U)$ is simple and there exists $\bar{\alpha}_{i}, i=1, \ldots, r$, and $\bar{\beta}_{j}$, $j=1, \ldots, s$, in $N /(0 ; U)$ such that

$$
\sum_{i, j} \bar{\alpha}_{i} \bar{\gamma}_{k} \bar{\beta}_{j}=\overline{1}
$$

(where $\overline{1}$ is the identity of $N /(0: U))$. By assumption (d), $(0: U)+(U: V)=N$ and there exists $\alpha_{i}$ and $\beta_{j}$ in $(U: V)$ such that $u \bar{\alpha}_{i}=u \alpha_{i}$ and $u \bar{\beta}_{j}=u \beta_{j}$ for $i=1, \ldots, r$, and $j=1, \ldots, s$. Let $\xi_{k}$ be as in 9.3 . Set

$$
\rho_{i j}=\left(\xi_{k}+\alpha_{i}\right) \gamma_{k} \beta_{j}
$$

for $i=1, \ldots, r$, and $j=1, \ldots, s$. Clearly each $\rho_{i j}$ is in $(U: V)$ which has an abelian additive group. The sum $\sum_{i, j} \rho_{i j}$ is therefore well defined. Take

$$
\delta_{k}=\xi_{k}+\sum_{i, j} \rho_{i j}
$$

Clearly $\delta_{k} \equiv 1 \bmod (U: V)$. Now for $u$ in $U$

$$
\left(v_{k}+u\right) \rho_{i j}=\left[v_{k} \xi_{k}+\left(v_{k}+u\right) \alpha_{i}\right] \gamma_{k} \beta_{j}
$$

and $v_{k} \xi_{k}=v_{k}+u^{\prime}\left(u^{\prime} \in U\right)$, since $\xi_{k} \equiv 1 \bmod (U: V)$. Thus

$$
\left(v_{k}+u\right) \rho_{i j}=\left[v_{k}+u^{\prime}+\left(v_{k}+u\right) \alpha_{i}\right] \gamma_{k} \beta_{j}
$$

and, since $u^{\prime}+\left(v_{k}+u\right) \alpha_{i}$ is in $U$, it follows from (i) of 9.5 that

$$
\left(v_{k}+u\right) \rho_{i j}=v_{k} \gamma_{k} \beta_{j}=v_{k} \rho_{i j}
$$

as can be seen on taking $u=0$. Thus for all $u$ in $U$

Hence (ii) follows. Now for $u$ in $U$

$$
\begin{aligned}
\left(v_{k}+u\right) \delta_{k} & =v_{k} \xi_{k}+\sum_{i, j} v_{k} \rho_{i j} \\
& =v_{k} \delta_{k} .
\end{aligned}
$$

$$
\begin{aligned}
u \rho_{i j} & =\left(u \xi_{k}+u \alpha_{i}\right) \gamma_{k} \beta_{j} \\
& =u \alpha_{i} \gamma_{k} \beta_{j}
\end{aligned}
$$

and by (1), $\sum_{i, j} u \rho_{i j}=u$. Thus for $u$ in $U$

$$
\begin{aligned}
u \delta_{k} & =u \xi_{k}+\sum_{i, j} u \rho_{i j} \\
& =u
\end{aligned}
$$

and (iii) follows. The lemma is proved. 
Lemma 9.7. Let $v_{i}, i \in I$, be a system of coset representatives of $V / U$ in $V$. Let $S$ be the subset of I consisting of all $s$ in I such that $v_{s} \equiv 0 \bmod C_{V}(U)$. If $S$ is a proper subset of $I$, then there exists $\rho$ in $N$ such that

(i) $\rho \equiv 1 \bmod (U: V)$;

(ii) $\left(v_{k}+u\right) \rho=v_{k} \rho$ for all $k$ in $I-S$ and $u$ in $U$; and

(iii) $u \rho=u$ for all $u$ in $U$.

Proof. Let $S_{1}$ be the set of all $\delta_{k}$ as in 9.6. Let $S_{2}$ be the set of all finite products of elements of $S_{1}$. Take $\rho$ in $S_{2}$ such that $\rho N$ is minimal (this is possible by 5.7). Clearly $\rho \equiv 1 \bmod (U: V)$ and $u \rho=u$ for all $u$ in $U$. Suppose for $i$ in $I-S$

$$
\left(v_{i}+u_{1}\right) \rho \neq v_{i} \rho
$$

for some $u_{1}$ in $U$. Consider $\rho \delta_{i}$ where $\delta_{i}$ is as in 9.6. Now for $u$ in $U$

$$
\left(v_{i}+u\right) \rho=v_{i}+u^{\prime}
$$

where $u^{\prime}$ is in $U$, because $\rho \equiv 1 \bmod (U: V)$. Thus

$$
\left(v_{i}+u\right) \rho \delta_{i}=v_{i} \delta_{i}=v_{i} \rho \delta_{i}
$$

for all $u$ in $U$. Hence

$$
\left(v_{i}+u\right) \rho \delta_{i} \beta=v_{i} \rho \delta_{i} \beta
$$

for all $\beta$ in $N$ and

$$
\left(v_{i}+u\right) \eta=v_{i} \eta
$$

for all $\eta$ in $\rho \delta_{i} N$. Now $\rho \delta_{i}$ is in $S_{2}$ and $\rho \delta_{i} N \leqq \rho N$. Since $\rho$ is in $\rho N$ we see from (1) that $\rho \delta_{i} N<\rho N$. This contradiction to the minimality of $\rho N$ establishes that $\rho$ satisfies (ii). The lemma is now proved.

Lemma 9.8. Let $v_{i}, i \in I$, be a system of coset representatives of $V / U$ in $V$. For $k$ in $I$ there exists $\pi_{k}$ in $N$ such that

(i) $\left(v_{k}+u_{1}\right) \pi_{k} \neq v_{k} \pi_{k}$ for some $u_{1}$ in $U$; and

(ii) $\left(v_{i}+u\right) \pi_{k}=v_{i} \pi_{k}$ for all $u$ in $U$ and $v_{i} \neq v_{k} \bmod C_{V}(U)$.

Proof. Let $v_{j}$ represent the coset $-v_{k}+U$. Let $\rho$ be as in 9.7. Take $\pi_{k}$ to be an element of $N$ such that

$$
\left(-v_{k}+v\right) \rho-\left(-v_{k}\right) \rho=v \pi_{k}
$$

for all $v$ in $V$. Now taking $v=v_{k}+u$ where $u$ is in $U$ we see

$$
\begin{aligned}
\left(v_{k}+u\right) \pi_{k} & =u \rho-\left(v_{j}\right) \rho \\
& =u-\left(v_{j}\right) \rho .
\end{aligned}
$$

Clearly $\left(v_{k}+u\right) \pi_{k} \neq v_{k} \pi_{k}$ for some $u$ in $U$ and (i) follows. Now if $i$ in $I$ is such that $v_{i}+u \neq v_{k} \bmod C_{V}(U)$ where $u$ is in $U$, then, since

$$
\left(v_{i}+u\right) \pi_{k}=\left(-v_{k}+v_{i}+u\right) \rho-\left(-v_{k}\right) \rho
$$


and

$$
-v_{k}+v_{i}+u=v_{r}+u^{\prime}
$$

for some $u^{\prime}$ in $U$ and $v_{r} \neq 0 \bmod C_{V}(U)$, it follows that

and the lemma follows.

$$
\begin{aligned}
\left(v_{i}+u\right) \pi_{k} & =v_{r} \rho-\left(-v_{k}\right) \rho \\
& =v_{i} \pi_{k}
\end{aligned}
$$

We are now in a position to state the main result of this section in the form of a theorem.

Theorem 9.9. If conditions $(a),(b),(c)$ and $(d)$ are satisfied, then the index of $C_{V}(U)$ in $V$ is finite.

Proof. We may assume that $C_{V}(U)<V$. Let $v_{i}, i \in I$, be a system of coset representatives of $V / U$ in $V$. We rename some of the indices in $I$ with the ordinals $1,2, \ldots$ in such a way that the $v_{b}, b$ an ordinal, are coset representatives of $C_{V}(U)$ in $V$.

Let $r$ be a positive integer and let $M_{r}$ be the set of all $\alpha$ in $N$ such that

$$
\left(v_{i}+u\right) \alpha=v_{i} \alpha
$$

for all $u$ in $U$ and all $i \in I$, where

$$
v_{i} \not \equiv v_{j} \bmod C_{V}(U)
$$

for any $j$ in $\{1, \ldots, r\}$. Clearly the $M_{n} r=1,2, \ldots$ are right $N$-subgroups of $N$ and

$$
M_{1} \leqq M_{2} \leqq M_{3} \leqq \ldots
$$

Let $\pi_{2}, \pi_{3}, \ldots$ be as in 9.8 . We have $\pi_{2}$ is in $M_{2}$ but not in $M_{1} ; \pi_{3}$ is in $M_{3}$ but not in $M_{2}$ etc. Thus, if the set of indexing ordinals is infinite, we have a contradiction to 5.7. It follows that the number of cosets of $C_{V}(U)$ in $V$ is finite and the theorem holds.

\section{Minimal condition and the near-ring generated by inner automorphisms}

In this section we prove that if the near-ring generated by the inner automorphisms of a group has minimal condition, then it is finite.

We state a lemma for tame $N$-groups where $N$ has minimal condition.

Lemma 10.1. Let $N$ be a near-ring with minimal condition on right ideals and $V a$ faithful tame $N$-group. If $U$ is a minimal $N$-subgroup of $V$ and $(U: V) \neq\{0\}$, then $(U: V)$ is a finite direct sum of right ideals $N$-isomorphic to $U$.

Proof. Since $V(U: V) \subseteq U$, it follows that $V(U: V) J(N)=\{0\},(U: V) J(N)=\{0\}$ and, by $5.3,(U: V) \leqq \operatorname{soc} N$. Now $\operatorname{soc} N$ is absolutely reducible in $N$. Thus $(U: V)$ is a direct sum of minimal right ideals (cf. $(2,15.2$, p. 86)). By minimal condition this direct sum is finite. Thus

$$
(U: V)=R_{1} \oplus \ldots \oplus R_{k}
$$

where $R_{i}, i=1, \ldots, k$, are minimal right ideals of $N$. For any $R_{i}, i$ in $\{1, \ldots, k\}$, there exists $v$ in $V$ such that $v R_{i} \neq\{0\}$. But since $R_{i} \leqq(U: V), v R_{i}=U$. Clearly $(0: v) \cap R_{i}=\{0\}$ and it follows that $R_{i}$ is $N$-isomorphic to $U$. The proof is complete. 
If $V$ is a group, then $I(V)$ will denote the near-ring generated by the inner automorphisms of $V$. In Section 6 we saw that $V$ is a compatible $I(V)$-group. Clearly the $I(V)$ subgroups of $V$ are precisely the normal subgroups.

Proposition 10.2 If $V$ is a group and $U$ a normal subgroup of $V$, then

$$
I(V / U) \cong I(V) /(U: V) \text {. }
$$

Let $N$ be a near-ring with a faithful compatible $N$-group $V$ and a minimal $N$-subgroup $U$. In Section 9 we were considering the situation where $(U: V) \not(0: U)$. These considerations are now required.

Lemma 10.3. Let $V$ be a group with a minimal normal subgroup $U$. If $I(V)$ has minimal condition on right ideals and $(U: V) \not(0: U)$, then $U$ is finite.

Proof. Suppose $U^{+}$is non-abelian. We have $U$ is a minimal compatible $I(V) /(0: U)$ group. In particular $U$ is 2-tame. Also for $u$ in $U, u N^{\prime}=U$ where $N^{\prime}=I(V) /(0: U)$. Thus $\left[N^{\prime}\right]^{+}$is non-abelian and a non-ring. Since $N^{\prime}$ has minimal condition, it follows by 8.4 that $N^{\prime} \cong M_{0}(U)$ and $U$ is finite (see $(6,7.19$, p. 198))

If $U^{+}$is abelian, then the inner automorphisms of $V$ distribute over $U$ and $I(V) /(0: U)\left(=N^{\prime}\right)$ is a ring and $U$ an $N^{\prime}$-module in the ring sense. The conditions (a) to (d) of Section 9 are satisfied. Thus the index $\left|V: C_{V}(U)\right|$ of $C_{V}(U)$ in $V$ is finite by 9.9. Now all $v$ in $V$ such that $[v, U]=\{0\}$ form a normal subgroup $C$ of $V$ containing $C_{V}(U)$. Thus $C_{V}(U) \leq C$ and, since $C$ is an $I(V)$-submodule, $C=C_{V}(U)$, and $|V: C|$ is finite. Let $v_{1}, \ldots, v_{n}$. be a system of coset representatives of $V / C$ in $V$. Let $u_{i}$ be a non-zero element of $U$. Clearly $U$ is the normal subgroup of $V$ generated by $u_{1}$, and thus $U$ is generated by all elements of the form

$$
v_{i}+v+u_{1}-v-v_{i}
$$

$i=1, \ldots, n$, with $v$ in $C$. Thus $U$ is generated by the elements of the form

$$
v_{i}+u_{1}-v_{i} \quad i=1, \ldots, n,
$$

of $V$. Hence $U$ is a finitely generated abelian group and is a finite direct sum $A_{1} \oplus \ldots \oplus A_{k}$ of cyclic groups. Also being a minimal normal subgroup of $V$, it is characteristically simple and therefore it is a finite elementary abelian $p$-group. The lemma is proved.

Theorem 10.4. Let $V$ be a group. If I( $V)$ has minimal condition on right ideals, then it is finite.

Proof. By 5.6 there exists a positive integer $k(V)$ and right ideals

$$
R_{0}=\{0\}<R_{1}<\ldots<R_{k(V)}=I(V)
$$

of $I(V)$ such that for $i=0, \ldots, k(V)-1, R_{i+1} / R_{i}$ is a minimal factor of $I(V)$. We proceed by induction on $k(V)$. If $k(V)=0$, then $I(V)=\{0\}$, the identity map of $V$ onto $V$ is zero and, $V=\{0\}$. It may therefore be assumed that $k(V)>0$ and that $I(V) \neq\{0\}$.

Out of all ideals of the form $(W: V)$ where $W$ is a submodule of $V$, choose one $(H: V)$ such that $(H: V)$ is minimal for being non-zero. We also assume that $H$ is minimal since, if 
$H_{i}, i \in I$, are such that $\left(H_{i}: V\right)=(H: V)$ and $W=\bigcap_{i \in I} H_{i}$, then $(W: V)=(H: V)$. Take $R$ to be a minimal right ideal of $I(V)$ contained in $(H: V)$. By $4.2, R$ is a minimal $I(V)$-group. Set

$$
K=\sum_{v \in V} v R
$$

For each $v$ in $V, v R=\{0\}$ or $v R$ is $I(V)$-isomorphic to $R$. Thus $K$ is, by 5.1 , absolutely reducible in $V$. Also $v R \leqq H, R \leqq(H: V)$, and we see that $K \leqq H$. But $R \leqq(K: V) \neq\{0\}$. From the minimality of $H, H=K$. From the absolute reducibility of $K, H=K_{0} \oplus K_{1}$, where $K_{0}$ is a minimal $I(V)$-subgroup of $V$. Set $Y=V / K_{1}$ and $X=K / K_{1}$. It follows that $X$ is a minimal submodule of $Y$ and by 10.2

$$
I(Y) /(X: Y) \cong I(Y / X) \cong I(V / K) \cong I(V) /(K: V)
$$

Now $Y=V / K_{1}$ and thus

$$
I(Y) \cong I(V) /\left(K_{1}: V\right)
$$

by 10.2 . Since, by the minimality of $K,\left(K_{1}: V\right)=\{0\}$ it follows that

$$
I(Y) \cong I(V) \text {. }
$$

But $(K: V)>\{0\}$ and $k(V)>k(V / K)$ so that by $(1)$ we may assume $I(Y) /(X: Y)$ is finite. From $(2)$ it will then follow that $I(V)$ is finite if it is shown that $(X: Y)$ is finite. By 10.1 this will follow if it is shown that $X$ is finite. It follows from 10.3 that we may assume $(X: Y) \leqq(0: X)$. Now $I(Y) /(0: X)(=N)$ has $X$ as a minimal $N$-group. Also

$$
[I(Y) /(X: Y)] /[(0: X) /(X: Y)] \cong N
$$

and $N$ is finite. Since for $x \neq 0$ in $X, x N=X$, it follows that $X$ is finite. The theorem is proved.

\section{Comments}

Although it has been assumed throughout that the tame $N$-groups considered are unitary this assumption is not always required. Indeed the results of Section 1 do not need the existence of an identity and the results of Sections 3 and 4 hold if $N$ has an identity although the $N$-groups considered may not be unitary.

One defect in the theory developed is that homomorphic images of tame (2-tame, compatible) near-rings need no longer be tame (2-tame, compatible). This defect can, to a certain extent; be overcome by generalising the notion of tame.

It seems hopeful that a worthwhile theory of tame near-rings with maximal condition on right ideals can be developed and that results resembling those due to Goldie (see (3, Ch. 3)) for rings, also hold.

In the considerations of Section 9 we dealt with the situation where $V$ was a faithful compatible $N$-group with a minimal submodule $U$ such that $(U: V) \not(0: U)$. In a more detailed study of the structure of a compatible near-ring $N$ with minimal condition this situation is of special importance. In this case the near-ring $N$ has a wreath product structure.

The final theorem (10.4) has as a consequence that if the near-ring generated by the 
inner automorphisms of a group $V$ has a minimal condition on right ideals, then $V$ is centre by finite. If maximal condition on right ideals is assumed it is still possible to obtain information about $V$. A question that would seem to be of importance in this direction is whether 9.9 holds with the weaker assumption of maximal condition.

As was noted in Section 6 the near-ring of zero-symmetric polynomial maps over an $\Omega$-group forms a compatible near-ring. Dr G. Baird and the author have considered this situation. It turns out that finite simple $\Omega$-groups are, apart from certain interesting exceptions, polynomially complete (cf. 8.4; for a definition of polynomial completeness see $(6,7.74, p$. 219) or (4) or (5)). The exceptions have been bound to be $\Omega$-groups bearing an interesting similarity to Lie-algebras. We hope to publish these results shortly.

The author wishes to thank Dr G. Baird for the interest he has taken in the writing of this paper and also the referee for his comments.

\section{REFERENCES}

(1) J. C. BeIdLeman, On the Theory of Radicals in d.g. near-rings II, Math. Ann. 173 (1967), 200-218.

(2) CURTIS and REINER, Representation Theory of Finite Groups and Associative Algebras (Interscience Publishers, New York, 1966).

(3) N. J. DivinSKY, Rings and Radicals (George Allen and Unwin Ltd, London, 1965).

(4) LAUSCH and NOBbaur, Algebra of Polynomials (North-Holland, Amsterdam, 1973).

(5) W. NÖBAUR, Funktionen auf kommutativen Ringen, Math. Ann. 147 (1962), 166-175.

(6) G. PILz, Near-rings (North-Holland, Amsterdam, 1977).

(7) S. D. ScoTt, Near-rings and near-ring modules (Doctoral dissertation, Australian National University, 1970).

Department of Mathematics

UNIVERSITY OF AUCKLAND

NEW ZEAL.AND 